\title{
Adaptive Fuzzy Fault-Tolerant Output Feedback Tracking Control of Uncertain Stochastic Nonlinear Systems with Unknown Time-Delay and Tracking Error Constrained
}

\author{
Shuai Sui, Shaocheng Tong, and Yongming Li \\ Department of Basic Mathematics, Liaoning University of Technology, Jinzhou, Liaoning 121000, China \\ Correspondence should be addressed to Shuai Sui; shuaisui2011@163.com
}

Received 21 July 2014; Accepted 2 September 2014; Published 5 November 2014

Academic Editor: Zhiguang Feng

Copyright (c) 2014 Shuai Sui et al. This is an open access article distributed under the Creative Commons Attribution License, which permits unrestricted use, distribution, and reproduction in any medium, provided the original work is properly cited.

\begin{abstract}
The problem of tracking error constrained adaptive fuzzy output feedback control is investigated for a class of single-input and single-output (SISO) stochastic nonlinear systems with actuator faults, unknown time-delay, and unmeasured states. The considered faults are modeled as both loss of effectiveness and lock-in-place. The fuzzy logic systems are used to approximate the unknown nonlinear functions, and a fuzzy adaptive observer is designed for estimating the unmeasured states. By transforming the tracking errors into new virtual error variables and based on backstepping recursive design technique, a new fuzzy adaptive output feedback control method is developed. It is shown that all the signals of the resulting closed-loop system are bounded in probability and the tracking error remains an adjustable neighborhood of the origin within the prescribed bounds. The simulation results are provided to show the effectiveness of the proposed approach.
\end{abstract}

\section{Introduction}

In recent years, the adaptive fuzzy or neural networks (NN) adaptive control design methods based on Itô's stochastic differential equation and backstepping design technique have been developed for some unknown stochastic nonlinear systems; see, for example, [1-11]. Authors in [1-3] proposed adaptive fuzzy state feedback control approaches for a class of SISO stochastic nonlinear systems with unknown virtual control gain function, unknown input saturation, and unknown dead-zone, respectively. Authors in [4] proposed an adaptive fuzzy backstepping control design method for a class of stochastic pure-feedback nonlinear systems with time-varying delays. Authors in [5] extended the results of [4] to a class of unknown stochastic pure-feedback nonlinear systems with unknown control directions, time-varying delays, and measurable states, while authors in [6-9] developed adaptive fuzzy output feedback controllers for SISO uncertain stochastic nonlinear systems with or without time-varying delays. And also, authors in $[10,11]$ proposed an adaptive fuzzy backstepping control approach for a class of uncertain stochastic nonlinear large-scale systems with immeasurable states.

However, the aforementioned control approaches assume that all the components of the considered stochastic nonlinear systems are in good operating conditions. In fact, the practical engineering always causes some faults, just like actuators and sensors, which often degrade the control performances and affect system stability or even catastrophic accidents. To handle the actuator faults involved in the considered nonlinear systems, many fault-tolerant control (FTC) design methods have been developed (see [12-17]). References $[12,13]$ proposed two adaptive fuzzy backstepping FTC control design methods for a class of unknown SISO nonlinear strict-feedback systems with both loss of effectiveness and lock-in-place actuator failures, in which they utilize fuzzy logic systems to approximate the unknown functions, and based on the backstepping recursive technique. The two methods not only guarantee the stability of the control system, but also achieve good robust performance. On the basis of the results of [12, 13], [14] proposed an adaptive fuzzy backstepping FTC control design method for a class 
of uncertain MIMO strict-feedback nonlinear systems and also prove the stability of the control system. However, the above mentioned FTC control schemes require that the state variables in the systems are measured directly. To solve the state's unmeasured problem, [15-17] developed two fuzzy adaptive output feedback FTC methods for a class of deterministic nonlinear systems and a class of stochastic nonlinear systems with actuator faults, respectively. It should be mentioned that although the aforementioned adaptive fuzzy output feedback control design methods have been developed for stochastic nonlinear systems, the unknown time-delay and tracking error constrained are neglected, which usually appear in many industrial control systems and often give rise to undesirable inaccuracy or even affect system stability.

It is worth pointing out that a design solution called prescribed performance control has been proposed in [18] for a class of feedback linearization nonlinear systems and was extended to the class of nonlinear systems in [19-21]. Its main idea is to introduce predefined performance bounds of the tracking errors and it is able to adjust control performance indices. However, to the author's best knowledge, by far, the prescribed performance design methodology has not been applied to unknown stochastic nonlinear strict-feedback systems with unknown functions, unknown time-delay, actuator faults, and immeasurable states, which is important and more practical, thus having motivated us for this study.

For the moment, there are few works to deal with such kinds of control systems in the literature at present stage, and few attempts have been made to pursue this novel idea. Motivated by the aforementioned observations, in this paper, authors proposed an adaptive fuzzy FTC method for a class of stochastic nonlinear systems with the actuator faults, immeasurable states, unknown time-delay, and tracking error constrained. Compared with the previous adaptive fuzzy control methods, the main advantages and contributions of the proposed control scheme are summarized as follows. (i) The problem of unmeasured state is solved by designing fuzzy state observer. (ii) The considered stochastic nonlinear systems include actuator failures and unknown time-delay. Consequently, the proposed adaptive controller not only can accommodate the actuator faults, but also has the robustness to the unknown time-delay. (iii) By introducing predefined performance, the proposed adaptive control method can not only ensure the closed-loop system to be stable, but also guarantee the tracking error to converge to a predefined arbitrarily small residual set.

\section{System Descriptions and Preliminaries}

2.1. Stochastic Nonlinear System Descriptions and Basic Assumptions. Consider the following strict-feedback stochastic nonlinear system:

$$
\begin{aligned}
d x_{i}=( & \left.x_{i+1}+f_{i}\left(\underline{x}_{i}\right)+h_{i}\left(\underline{x}_{i}\left(t-\tau_{i}(t)\right)\right)+\Delta_{i}(x, t)\right) d t \\
& +g_{i}(x) d w, \quad i=1, \ldots, n-1
\end{aligned}
$$

$$
\begin{aligned}
d x_{n}= & \left(\omega^{T} u+f_{n}\left(\underline{x}_{n}\right)+h_{n}\left(\underline{x}_{n}\left(t-\tau_{n}(t)\right)\right)+\Delta_{n}(x, t)\right) d t \\
& \quad+g_{n}(x) d w \\
y= & x_{1},
\end{aligned}
$$

where $\underline{x}_{i}=\left[x_{1}, x_{2}, \ldots, x_{i}\right]^{T} \in \mathfrak{R}^{i}, i=1,2, \ldots, n(x=$ $\left.\underline{x}_{n}\right)$, are the states, $y \in \Re$ is the system output, and $u=$ $\left[u_{1}, u_{2}, \ldots, u_{m}\right]^{T} \in \mathfrak{R}^{m}$ is the input vector of the system; that is, $u_{i}$ is the output of the $i$ th actuator, $\emptyset=\left(\varrho_{1}, \varrho_{2}, \ldots, \emptyset_{m}\right)^{T} \in$ $\mathfrak{R}^{m}, \emptyset_{j}, j=1,2, \ldots, m$, are known constant vectors. $f_{i}\left(\underline{x}_{i}\right)$, $i=1,2, \ldots, n$, are unknown continuous nonlinear functions; $\Delta_{i}(x, t)$ is unknown bounded external disturbances satisfying $\Delta_{i}(x, t) \leq \Delta_{i}^{*}(x, t)$, with $\Delta_{i}^{*}(x, t)$ being unknown constant. $g_{i}(x)$ is the uncertain function, $h_{i}\left(\underline{x}_{i}\left(t-\tau_{i}(t)\right)\right), 1 \leq i \leq n$, are unknown smooth nonlinear functions, and $\tau_{i}(t)$ is an unknown time-delay with $\dot{\tau}_{i}(t) \leq \tau^{*} \leq 1,1 \leq i \leq n . w \in \Re$ is an independent $r$-dimensional Wiener process defined on a complete probability space with the incremental covariance $E\left\{d w \cdot d w_{j}^{T}\right\}=\sigma(t) \sigma(t)^{T} d t$. In this paper, it is assumed that only output $y$ is available for measurement.

The actuator faults considered in this paper are both lockin-place and loss of effectiveness, which were defined by [1217] as follows.

Lock-in-Place Model ([12-14]). Consider the following:

$$
u_{i}(t)=\bar{u}_{i}, \quad t \geq t_{i}, \quad i \in\left\{j_{1}, j_{2}, \ldots, j_{p}\right\} \subset\{1,2, \ldots, m\} .
$$

Loss of Effectiveness Model ([12-14]). Consider the following:

$$
\begin{gathered}
u_{i}(t)=\eta_{i} v_{i}(t), \quad t \geq t_{i}, \quad i \in \overline{\left\{j_{1}, j_{2}, \ldots, j_{p}\right\}} \cap\{1,2, \ldots, m\} \\
\eta_{i} \in\left[\underline{\eta}_{i}, 1\right], \quad 0<\underline{\eta}_{i} \leq 1,
\end{gathered}
$$

where $\bar{u}_{i}$ is the constant value where the actuator stuck at and $t_{i}$ are the time instants when some faults take place. $\eta_{i}$ is the still effective proportion of the actuator after losing some effectiveness, and $\underline{\eta}_{i}$ is the lower bound of $\eta_{i}$. When $\underline{\eta}_{i}$ is 1 , the corresponding actuator is normal (no fault happens).

Taking the actuator faults (2) and (3) into account, the input vector can be written as

$$
u(t)=\rho v(t)+\sigma(\bar{u}-\rho v(t)),
$$

where $v(t)=\left[v_{1}(t), v_{2}(t), \ldots, v_{m}(t)\right]^{T}$ is the applied control vector. $\bar{u}=\left[\bar{u}_{1}, \bar{u}_{2}, \ldots, \bar{u}_{m}\right]^{T}$ with $\bar{u}_{j}, j=1,2, \ldots, m$, are constant values, and

$$
\begin{aligned}
& \rho=\operatorname{diag}\left\{\rho_{1}, \rho_{2}, \ldots, \rho_{m}\right\}, \\
& \sigma=\operatorname{diag}\left\{\sigma_{1}, \sigma_{2}, \ldots, \sigma_{m}\right\}
\end{aligned}
$$

with

$$
\sigma_{j}= \begin{cases}1, & \text { if the } i \text { th actuator fails as }(2), \text { that is, } u_{j}=\bar{u}_{j} \\ 0, & \text { otherwise. }\end{cases}
$$


Our control objective is to design an adaptive fuzzy output feedback FTC scheme for the system (1) to ensure that all the signals in the closed-loop system are bounded in probability and the output $y(t)$ can track the given reference signal $y_{r}(t)$ with the given prescribed performance bounds. To this end, the following assumptions are made for the proposed control designs.

Assumption 1 (see [4-6]). Nonlinear function $h_{i}$ satisfies the following inequalities for $i=1,2, \ldots, n$ :

$$
\left|h_{i}\left(\underline{x}_{i}(t)\right)\right|^{2} \leq \chi_{1}(t) H_{i 2}\left(\chi_{1}(t)\right)+\bar{h}_{i 2}\left(y_{r}(t)\right)+\Phi_{i},
$$

where $H_{i 2}(\cdot)$ is a known function, $\bar{h}_{i 2}$ is bounded function with $\bar{h}_{i 2}(0)=0$, and $\varrho_{i}$ is positive scalars; $\chi_{1}$ is referred to as a virtual error variable, which will be designed later.

Assumption 2. Assume that functions $f_{i}(\cdot)$ satisfy the global Lipschitz condition; that is, there exist known constants $m_{i}$, $i=1,2, \ldots, n$ such that, for all $X_{1}, X_{2} \in \mathfrak{R}^{i}$, the following inequalities hold:

$$
\left|f_{i}\left(X_{1}\right)-f_{i}\left(X_{2}\right)\right| \leq m_{i}\left\|X_{1}-X_{2}\right\|,
$$

where $\|X\|$ denotes the 2-norm of a vector $X$.

Assumption 3 (see [12-17]). System (1) requires that for any up to $m-1$ actuators which fail as (2) and the other(s) which may lose effectiveness as (3), the resulting system can still achieve the desired control objective.

Assumption 4 (see [22]). The disturbance covariance $g^{T} \sigma \sigma^{T} g=\overline{\sigma \sigma}^{T}$ is bounded, where $g=\left[g_{1}, \ldots, g_{n}\right]^{T}$ is $r \times n$ matrix-valued function.

2.2. Fuzzy Logic Systems. A fuzzy logic system (FLS) consists of four parts: the knowledge base, the fuzzifier, the fuzzy inference engine working on fuzzy rules, and the defuzzifier. The knowledge base for FLS comprises a collection of fuzzy If-then rules of the following form:

$$
\begin{aligned}
& R^{l}: \text { If } x_{1} \text { is } F_{1}^{l} \text { and } x_{2} \text { is } F_{2}^{l} \text { and } \ldots \text { and } x_{n} \text { is } F_{n}^{l} \text {, } \\
& \text { Then } y \text { is } G^{l}, \quad l=1,2, \ldots, N \text {, }
\end{aligned}
$$

where $x=\left(x_{1}, \ldots, x_{n}\right)^{T}$ and $y$ are the fuzzy logic system input and output, respectively. Fuzzy sets $F_{i}^{l}$ and $G^{l}$ associate with the fuzzy functions $\mu_{F_{i}^{l}}\left(x_{i}\right)$ and $\mu_{G^{l}}(y)$, respectively. $N$ is the rule number of IF-THEN.

Through singleton function, center average defuzzification, and product inference [23], the fuzzy logic system can be expressed as

$$
y(x)=\frac{\sum_{l=1}^{N} \bar{y}_{l} \prod_{i=1}^{n} \mu_{F_{i}^{l}}\left(x_{i}\right)}{\sum_{l=1}^{N}\left[\prod_{i=1}^{n} \mu_{F_{i}^{l}}\left(x_{i}\right)\right]},
$$

where $\bar{y}_{l}=\max _{y \in R} \mu_{G^{l}}(y)$.
Define the fuzzy basis functions as

$$
\varphi_{l}=\frac{\prod_{i=1}^{n} \mu_{F_{i}^{l}}\left(x_{i}\right)}{\sum_{l=1}^{N}\left(\prod_{i=1}^{n} \mu_{F_{i}^{l}}\left(x_{i}\right)\right)} .
$$

Denoting $\theta^{T}=\left[\bar{y}_{1}, \bar{y}_{2}, \ldots, \bar{y}_{N}\right]=\left[\theta_{1}, \theta_{2}, \ldots, \theta_{N}\right]$ and $\varphi(x)=\left[\varphi_{1}(x), \ldots, \varphi_{N}(x)\right]^{T}$, then fuzzy logic system (10) can be rewritten as

$$
y(x)=\theta^{T} \xi(x) .
$$

Lemma 5 (see [23]). Let $f(x)$ be a continuous function defined on a compact set $\Omega$. Then for any constant $\varepsilon>0$, there exists a fuzzy logic system (14) such that

$$
\sup _{x \in \Omega}\left|f(x)-\theta^{T} \varphi(x)\right| \leq \varepsilon .
$$

2.3. Performance Function and Error Transformation. This section introduces preliminary knowledge on the prescribed performance concepts reported in $[20,21]$. According to [20, 21], the prescribed performance is achieved by ensuring that tracking error $z_{1}(t)=y-y_{r}$ evolves strictly within predefined decaying bounds as follows:

$$
-\delta_{1 \min } \mu_{1}(t)<z_{1}(t)<\delta_{1 \max } \mu_{1}(t), \quad \forall t \geq 0,
$$

where $\delta_{1 \min }$ and $\delta_{1 \max }$ are design constants and the performance functions $\mu_{1}(t)$ are bounded and strictly positive decreasing smooth functions with the property $\lim _{t \rightarrow \infty} \mu_{1}(t)=\mu_{1, \infty} ; \mu_{1, \infty}>0$ is a constant. The performance functions are chosen as the exponential form $\mu_{1}(t)=\left(\mu_{1,0}-\mu_{1, \infty}\right) e^{-n_{1} t}+\mu_{1, \infty}$, where $n_{1}, \mu_{1,0}$, and $\mu_{1, \infty}$ are strictly positive constants, $\mu_{1,0}>\mu_{1, \infty}$, and $\mu_{1,0}=\mu_{1}(0)$ is selected such that $-\delta_{1 \min } \mu_{1}(0)<z_{1}(0)<\delta_{1 \max } \mu_{1}(0)$ is satisfied. Furthermore, the maximum overshoot of $z_{1}(t)$ is prescribed less than $\max \left\{\delta_{1 \min } \mu_{1}(0), \delta_{1 \max } \mu_{1}(0)\right\}$. Therefore, choosing the performance function $\mu_{1}(t)$ and the constants $\delta_{1 \text { min }}$ and $\delta_{1 \max }$ appropriately determines the performance bounds of the error $z_{1}(t)$.

To represent (14) by an equality form, we employ an error transformation as

$$
z_{1}=\mu_{1}(t) \Phi_{1}\left(\zeta_{1}(t)\right), \quad \forall t \geq 0,
$$

where $\Phi_{1}\left(\zeta_{1}\right)=\left(\delta_{1 \max } e^{\zeta_{1}}-\delta_{1 \min } e^{-\zeta_{1}}\right) /\left(e^{\zeta_{1}}+e^{-\zeta_{1}}\right), \zeta_{1}(t)=$ $\Phi^{-1}\left(z_{1}(t) / \mu_{1}(t)\right)=(1 / 2) \ln \left(\left(\Phi_{1}-\delta_{1 \min }\right) /\left(\delta_{1 \max }-\Phi_{1}\right)\right)$, and $\dot{\zeta}_{1}(t)=p_{1}\left(\dot{z}_{1}-\left(\dot{\mu}_{1} z_{1} / \mu_{1}\right)\right)$, with $p_{1}=\left(1 / 2 \mu_{1}\right)\left[1 /\left(\Phi_{1}+\delta_{1 \min }\right)-\right.$ $\left.1 /\left(\Phi_{1}-\delta_{1 \max }\right)\right]$.

For the output feedback control design of the nonlinear system, we design the following state transformation:

$$
\chi_{1}(t)=\zeta_{1}(t)-\frac{1}{2} \ln \frac{\delta_{1 \min }}{\delta_{1 \max }} .
$$

And the transformation state dynamics is

$$
\dot{\chi}_{1}(t)=p_{1}\left(\dot{z}_{1}-\frac{\dot{\mu}_{1} z_{1}}{\mu_{1}}\right) .
$$




\section{Fuzzy State Observer Design}

Note that, in the system (1), the states $x_{2}, \ldots, x_{n}$ are not measured directly; thus a state observer should be established to obtain the estimations of $x_{2}, \ldots, x_{n}$.

Rewrite (1) in the following form:

$$
\begin{aligned}
d x= & \left(A x+K y+\sum_{i=1}^{n} B_{i}\left(f_{i}\left(\underline{x}_{i}\right)+\Delta_{i}+h_{i}\right)+B \omega^{T} u\right) d t \\
& +g(x) d w \\
= & \left(A x+K y+\sum_{i=1}^{n} B_{i}\left(f_{i}\left(\underline{x}_{i}\right)+\Delta f_{i}+\Delta_{i}+h_{i}\right)+B \omega^{T} u\right) d t \\
& +g(x) d w,
\end{aligned}
$$$$
y=C x
$$

where $K=\left[k_{1}, k_{2}, \ldots, k_{n}\right]^{T}, B_{i}=\left[\begin{array}{lllll}0 & \cdots & 1 & \cdots & 0\end{array}\right]^{T}, B=$ $\left[\begin{array}{lllll}0 & \cdots & 0 & \cdots & 1\end{array}\right]^{T}, g(x)=\left[g_{1}, \ldots, g_{n}\right]^{T}, C=\left[\begin{array}{lllll}1 & \cdots & 0 & \cdots & 0\end{array}\right], \Delta f_{i}=$ $f_{i}\left(\underline{x}_{i}\right)-f_{i}\left(\underline{x}_{i}\right)$, and $A=\left[\begin{array}{cc}-k_{1} & \\ \vdots & I \\ -k_{n} & 0 \cdots 0\end{array}\right]$.

Given a positive definite matrix $Q=Q^{T}>0$ and an appropriate constant $\beta>0$, choose vector $K$ satisfying the following matrix inequality:

$$
A^{T} P+P A+\left(4+\frac{n}{2}+\beta \sum_{i=1}^{n} m_{i}^{2}\right) I+\frac{1}{\beta} P P^{T}<-Q
$$

where $P=P^{T}>0$ is a positive definite matrix.

The matrix inequality (19) is widely used in $T-S$ fuzzy system control design. To solve positive definite matrix and vector $K$ from (19), the matrix $A=\bar{A}+K D$ need be decomposed into $\bar{A}=\left[\begin{array}{cc}0 & I_{n-1} \\ 0 & 0\end{array}\right]$ and $D=\left[\begin{array}{cccc}-1 & 0 & \cdots & 0\end{array}\right]$.

Then, (19) can be transformed into a standard linear matrix inequality (LMIs):

$$
\left[\begin{array}{cc}
E & P \\
P & -\beta I
\end{array}\right]<0
$$

where $E=\left(\bar{A}^{T} P+P \bar{A}+D^{T} N^{T}+N D\right)+\left(4+(n / 2)+\beta \sum_{i=1}^{n} m_{i}^{2}\right) I+$ $Q, N=P K$. By using LMIs (20), we can obtain $P$ and $N$. Furthermore, the matrix $K$ can be computed as $K=P^{-1} N$ [4-6].

By Lemma 5, we can assume that nonlinear terms $f_{i}\left(\underline{x}_{i}\right)$, $i=1,2, \ldots, n$, in (14) can be approximated by the following fuzzy logic systems:

$$
\widehat{f}_{i}\left(\underline{\hat{x}}_{i} \mid \theta_{i}\right)=\theta_{i}^{T} \varphi\left(\underline{x}_{i}\right),
$$

where $\underline{x}_{i}=\left(\widehat{x}_{1}, \widehat{x}_{2}, \ldots, \widehat{x}_{i}\right)^{T}$ is the estimation of $\underline{x}_{i}=$ $\left(x_{1}, x_{2}, \ldots, x_{i}\right)^{T}$.

Define the optimal parameter vectors $\theta_{i}^{*}$ as

$$
\theta_{i}^{*}=\underset{\theta_{i} \in \Omega_{i}}{\operatorname{argmin}}\left[\sup _{\overline{\bar{x}}_{i} \in U_{i}}\left|\widehat{f}_{i}\left(\underline{\hat{x}}_{i} \mid \theta_{i}\right)-f_{i}\left(\underline{\widehat{x}}_{i}\right)\right|\right],
$$

where $\Omega_{i}$ and $U_{i}$ are bounded compact sets for $\theta_{i}$ and $\underline{x}_{i}$, respectively. Also, the fuzzy minimum approximation error $\varepsilon_{i}$ is defined as

$$
\varepsilon_{i}=f_{i}\left(\underline{\underline{x}}_{i}\right)-\widehat{f}_{i}\left(\underline{\underline{x}}_{i} \mid \theta_{i}^{*}\right),
$$

where $\varepsilon_{i}$ satisfies $\left|\varepsilon_{i}\right| \leq \varepsilon_{i}^{*}$, with $\varepsilon_{i}^{*}$ being a positive constant.

The state observer for (18) is designed as

$$
\begin{gathered}
\dot{\hat{x}}=A \widehat{x}+K y+\sum_{i=1}^{n} B_{i} \widehat{f}_{i}\left(\underline{x}_{i} \mid \theta_{i}\right)+B \omega^{T} u, \\
\hat{y}=C \widehat{x} .
\end{gathered}
$$

Let $e=x-\widehat{x}$ be the observer error vector. Then from (18) and (24), we have the observer error equation

$$
\begin{aligned}
d e= & \left(A e+\sum_{i=1}^{n} B_{i}\left[f_{i}\left(\underline{\widehat{x}}_{i}\right)-\widehat{f}_{i}\left(\underline{\widehat{x}}_{i} \mid \theta_{i}\right)+\Delta f_{i}+\Delta_{i}+h_{i}\right]\right) d t \\
& +g(x) d w \\
= & \left(A e+\sum_{i=1}^{n} B_{i}\left[\widetilde{\theta}_{i}^{T} \varphi_{i}\left(\underline{\hat{x}}_{i}\right)+\varepsilon_{i}+\Delta f_{i}+\Delta_{i}+h_{i}\right]\right) d t \\
& +g(x) d w
\end{aligned}
$$

where $\widetilde{\theta}_{i}=\theta_{i}^{*}-\theta_{i}$ is the parameter error vector.

Theorem 6. Consider the following Lyapunov candidate $V_{0}$ for the observer error system (25):

$$
V_{0}=e^{T} P e .
$$

Then $\ell V_{0}$ is bounded by

$$
\begin{aligned}
\ell V_{0} \leq & e^{T}\left(A^{T} P+P A\right) e+\left(4+\|P\|^{2} \sum_{i=1}^{n} m_{i}^{2}\right)\|e\|^{2} \\
& +\sum_{i=1}^{n} \widetilde{\theta}_{i}^{T} \widetilde{\theta}_{i}+d_{0} \\
& +\sum_{i=1}^{n} \chi_{1}\left(t-\tau_{i}(t)\right) H_{i 2}\left(\chi_{1}\left(t-\tau_{i}(t)\right)\right)
\end{aligned}
$$

where $d_{0}=(1 / 2)\left|\overline{\sigma \sigma}^{T}\right|^{2}+\|P\|^{2}\left(1 / 2+\left\|\varepsilon^{*}\right\|^{2}+\left\|\Delta^{*}\right\|^{2}\right)+\sum_{i=1}^{n} d_{i}^{*}$.

Proof. See the Appendix.

\section{Fault-Tolerant Control Design and Stability Analysis}

In this section, an adaptive fuzzy fault-tolerant control scheme will be developed by using the above fuzzy state observer and the backstepping technique [24, 25], and the stability of the closed-loop system will be given. 
According to [12-14], we choose the following special control structure:

$$
v_{j}=b_{j}\left(\widehat{x}_{n}\right) u_{0},
$$

where $0 \leq \underline{b}_{j} \leq b_{j}\left(\widehat{x}_{n}\right) \leq \bar{b}_{j}$, for all $\underline{x}_{n} \in \Omega_{n} \subset R^{n}$, $j=1,2, \ldots, m ; \underline{b}_{j}$ and $\bar{b}_{j}$ are the lower and upper bounds of $b_{j}\left(\widehat{x}_{n}\right)$, respectively. $u_{0}$ is the final controller to be designed by the backstepping technique as follows.

The $n$-step adaptive fuzzy output feedback backstepping FTC design is based on the following changes of coordinates:

$$
\begin{gathered}
\chi_{1}=\chi_{1}, \\
\chi_{i}=\widehat{x}_{i}-\alpha_{i-1}, \quad i=2, \ldots, n,
\end{gathered}
$$

where $\alpha_{i-1}$ are the virtual control functions to be designed later.

Step 1. From (1), (17), and (29), one has

$$
\begin{aligned}
d x_{1}= & p_{1}\left(x_{2}+f_{1}\left(x_{1}\right)+\Delta_{1}(x, t)\right. \\
& \left.+h_{1}\left(x_{1}\left(t-\tau_{1}(t)\right)\right)-\dot{y}_{r}-\frac{\dot{\mu}_{1} z_{1}}{\mu_{1}}\right) d t \\
& +g_{1}(x) d w \\
= & p_{1}\left(\widehat{x}_{2}+e_{2}+\theta_{1}^{T} \varphi\left(\widehat{x}_{1}\right)+\widetilde{\theta}_{1}^{T} \varphi\left(\widehat{x}_{1}\right)\right. \\
& +\varepsilon_{1}+\Delta f_{1}+\Delta_{1}(x, t) \\
& \left.+h_{1}\left(x_{1}\left(t-\tau_{1}(t)\right)\right)-\dot{y}_{r}-\frac{\dot{\mu}_{1} z_{1}}{\mu_{1}}\right) d t+g_{1}(x) d w .
\end{aligned}
$$

By using (21), (24) can be rewritten as

$$
\begin{aligned}
d \chi_{1}=p_{1}( & \chi_{2}+\alpha_{1}+e_{2}+\theta_{1}^{T} \varphi\left(\widehat{x}_{1}\right)+\widetilde{\theta}_{1}^{T} \varphi\left(\widehat{x}_{1}\right) \\
& +\varepsilon_{1}+\Delta f_{1}+\Delta_{1}(x, t) \\
& \left.+h_{1}\left(x_{1}\left(t-\tau_{1}(t)\right)\right)-\dot{y}_{r}-\frac{\dot{\mu}_{1} z_{1}}{\mu_{1}}\right) d t+g_{1}(x) d w .
\end{aligned}
$$

Consider the following Lyapunov function candidate:

$$
V_{1}=V_{0}+\frac{\chi_{1}^{2}}{2}+\frac{1}{2 \gamma_{1}} \widetilde{\theta}_{1}^{T} \widetilde{\theta}_{1}+W_{1}+\bar{V}_{1}
$$

where $\gamma_{1}>0$ is a design parameter. $W_{1}=\left(e^{r \tau} / 2(1-\right.$ $\left.\left.\tau^{*}\right)\right) e^{-r t} \int_{t-\tau_{1}(t)}^{t} e^{r \zeta} \chi_{1}(\zeta) H_{12}\left(\chi_{1}(\zeta)\right) d \zeta$ and $\bar{V}_{1}=\left(\beta e^{r \tau} /(1-\right.$ $\left.\left.\tau^{*}\right)\right) \sum_{i=1}^{n} e^{-r t} \int_{t-\tau_{i}(t)}^{t} e^{r \zeta} \chi_{1}(\zeta) H_{i 2}\left(\chi_{1}(\zeta)\right) d \zeta$ in which $r>0$ and $\tau \geq 0 \max \left\{\tau_{1}(t), \ldots, \tau_{n}(t)\right\}$ are known constants.
From (25) and (27), the infinitesimal generator of $V_{1}$ satisfies

$$
\begin{aligned}
\ell V_{1}= & \ell V_{0}+\chi_{1} p_{1} \\
\times & \left(\chi_{2}+\alpha_{1}+e_{2}+\theta_{1}^{T} \varphi\left(\widehat{x}_{1}\right)\right. \\
& +\widetilde{\theta}_{1}^{T} \varphi\left(\widehat{x}_{1}\right)+\varepsilon_{1}+\Delta f_{1}+\Delta_{1}(x, t) \\
& \left.+h_{1}\left(x_{1}\left(t-\tau_{1}(t)\right)\right)-\dot{y}_{r}-\frac{\dot{\mu}_{1} z_{1}}{\mu_{1}}\right) \\
& +\frac{1}{2} g_{1}^{T} \sigma \sigma^{T} g_{1}-\frac{1}{\gamma_{1}} \widetilde{\theta}_{1}^{T} \dot{\theta}_{1}+\dot{W}_{1} .
\end{aligned}
$$

By using Young's inequality, one can obtain

$$
\begin{aligned}
& \chi_{1} p_{1}\left(\chi_{2}+e_{2}+\varepsilon_{1}+\Delta_{1}(x, t)\right) \\
& \leq 2 \chi_{1}^{2} p_{1}^{2}+\frac{1}{4}+\frac{1}{4} \chi_{2}^{4}+\frac{1}{2}\|e\|^{2}+\frac{1}{2} \Delta_{1}^{* 2}+\frac{1}{2} \varepsilon_{1}^{* 2} .
\end{aligned}
$$

By using Assumption 4 and Young's inequality, one has

$$
\begin{aligned}
& \chi_{1} p_{1} h_{1}\left(\underline{x}_{1}\left(t-\tau_{1}(t)\right)\right) \\
& \leq \frac{1}{2} \chi_{1}^{2} p_{1}^{2}+\frac{1}{2} \chi_{1}\left(t-\tau_{1}(t)\right) H_{12}\left(\chi_{1}\left(t-\tau_{1}(t)\right)\right)+d_{1}^{*},
\end{aligned}
$$

where $d_{1}^{*}$ is a constant with $d_{1}^{*} \geq \bar{h}_{12}\left(y_{r}\left(t-\tau_{1}(t)\right)\right)+(1 / 2) \varrho_{1}$.

By using Assumption 4 and Young's inequality, one can obtain the following inequality:

$$
\frac{1}{2} g_{1}^{T} \sigma \sigma^{T} g_{1} \leq \frac{1}{4}+\frac{1}{4}|\overline{\sigma \sigma}|^{T} .
$$

It is noticed that

$$
\begin{gathered}
\dot{W}_{1} \leq-r W_{1}+\frac{e^{r \tau}}{2\left(1-\tau^{*}\right)} \chi_{1} H_{12}\left(\chi_{1}\right) \\
-\frac{1}{2} \chi_{1}\left(t-\tau_{1}(t)\right) H_{12}\left(\chi_{1}\left(t-\tau_{1}(t)\right)\right), \\
\dot{\bar{V}}_{1} \leq_{1}-r \bar{V}_{1}-\beta \sum_{i=1}^{n} \chi_{1}\left(t-\tau_{i}(t)\right) H_{i 2}\left(\chi_{1}\left(t-\tau_{i}(t)\right)\right) .
\end{gathered}
$$

Substituting (29)-(32) into (28) yields

$$
\begin{aligned}
\ell V_{1}= & \chi_{1} p_{1}\left(\alpha_{1}+\theta_{1}^{T} \varphi\left(\widehat{x}_{1}\right)+\frac{5}{2} \chi_{1} p_{1}\right. \\
& \left.+\frac{e^{r \tau}}{2 p_{1}\left(1-\tau^{*}\right)} H_{12}\left(\chi_{1}\right)-\dot{y}_{r}-\frac{\dot{\mu}_{1} z_{1}}{\mu_{1}}\right) \\
& +\frac{1}{4} \chi_{2}^{4}+e^{T}\left(A^{T} P+P A\right) e \\
& +\left(4+\frac{1}{2}+\|P\|^{2} \sum_{i=1}^{n} m_{i}^{2}\right)\|e\|^{2} \\
& -\widetilde{\theta}_{1}^{T}\left(\frac{\dot{\theta}_{1}}{\gamma_{1}}-\varphi\left(\widehat{x}_{1}\right) \chi_{1}\right)+\sum_{i=1}^{n} \widetilde{\theta}_{i}^{T} \widetilde{\theta}_{i}+d_{1}-r W_{1},
\end{aligned}
$$


where $d_{1}=d_{0}+d_{1}^{*}+(1 / 2) \varepsilon_{1}^{* 2}+(3 / 4)+\left.(1 / 4)|\overline{\sigma \sigma}|^{T}\right|^{2}+(1 / 2) \Delta_{1}^{* 2}+$ $(1 / 2) \varepsilon_{1}^{* 2}$.

Choose the intermediate control function, $\alpha_{1}$, and the adaptation law for $\theta_{1}$ as follows:

$$
\begin{gathered}
\alpha_{1}=-c_{1} \chi_{1} p_{1}-\theta_{1}^{T} \varphi\left(\widehat{x}_{1}\right)-\frac{5}{2} \chi_{1} p_{1} \\
-\frac{n e^{r \tau}}{2 p_{1}\left(1-\tau^{*}\right)} H_{12}\left(\chi_{1}\right)+\dot{y}_{r}+\frac{\dot{\mu}_{1} z_{1}}{\mu_{1}} \\
-\frac{\beta e^{r \tau}}{p_{1}\left(1-\tau^{*}\right)} \sum_{i=1}^{n} H_{i 2}\left(\chi_{1}\right), \\
\dot{\theta}_{1}=\gamma_{1} \varphi\left(\widehat{x}_{1}\right) \chi_{1}-\sigma_{1} \theta_{1},
\end{gathered}
$$

where $c_{1}>0$ and $\sigma_{1}>0$ are design parameters.

Substituting (34)-(36) into (33), one has

$$
\begin{aligned}
\ell V_{1}= & e^{T}\left(A^{T} P+P A\right) e+\left(4+\frac{1}{2}+\|P\|^{2} \sum_{i=1}^{n} m_{i}^{2}\right)\|e\|^{2} \\
& -c_{1} p_{1}^{2} \chi_{1}^{2}+\frac{1}{4} \chi_{2}^{4}+\frac{\sigma_{1}}{\gamma_{1}} \widetilde{\theta}_{1}^{T} \theta_{1} \\
& +\sum_{i=1}^{n} \widetilde{\theta}_{i}^{T} \widetilde{\theta}_{i}-\frac{(n-1) \chi_{1} e^{r \tau}}{2\left(1-\tau^{*}\right)} H_{12}\left(\chi_{1}\right)+d_{1}-r W_{1} .
\end{aligned}
$$

Step $i(2 \leq i \leq n-1)$. From (14) and (21) and similar to Step 1, one has

$$
\begin{aligned}
d \chi_{i}= & \left(\widehat{x}_{i+1}+\widehat{f}_{i}\left(\widehat{x}_{i} \mid \theta_{i}\right)+k_{i}\left(x_{1}-\widehat{x}_{1}\right)\right) d t-\sum_{j=1}^{i-1} \frac{\partial \alpha_{i-1}}{\partial \theta_{j}} \dot{\theta}_{j} d t \\
& -\frac{\partial \alpha_{i-1}}{\partial y} d y-\sum_{j=1}^{i-1} \frac{\partial \alpha_{i-1}}{\partial \widehat{x}_{j}} d \widehat{x}_{j} \\
= & \left(\alpha_{i}+\chi_{i+1}+H_{i}-\frac{\partial \alpha_{i-1}}{\partial y}\right. \\
& \times\left(e_{2}+\widetilde{\theta}_{1}^{T} \varphi\left(\widehat{x}_{1}\right)+\varepsilon_{1}+\Delta_{1}(x, t)+h_{1}\left(\underline{x}_{1}\left(t-\tau_{1}(t)\right)\right)\right. \\
& \left.\left.\quad-\frac{1}{2} \frac{\partial^{2} \alpha_{i-1}}{\partial y^{2}} g_{1}^{T} \sigma \sigma^{T} g_{1}\right)\right) d t-\frac{\partial \alpha_{i-1}}{\partial y} g_{1}(x) d w
\end{aligned}
$$

where $H_{i}=\theta_{i}^{T} \varphi\left(\underline{\widehat{x}}_{i}\right)+\left(k_{i}-\sum_{j=1}^{i-1}\left(\partial \alpha_{i-1} / \partial \widehat{x}_{j}\right) k_{j}\right)\left(x_{1}-\right.$ $\left.\widehat{x}_{1}\right)-\sum_{j=1}^{i-1}\left(\partial \alpha_{i-1} / \partial \theta_{j}\right) \dot{\theta}_{j}-\left(\partial \alpha_{i-1} / \partial y\right)\left(\widehat{x}_{2}+\theta_{1}^{T} \varphi\left(\widehat{x}_{1}\right)\right)-$ $\sum_{j=1}^{i-1}\left(\partial \alpha_{i-1} / \partial \widehat{x}_{j}\right)\left(\widehat{x}_{j+1}+\widehat{f}_{j}\left(\underline{\underline{x}}_{j} \mid \theta_{j}\right)\right)$.

Consider the following Lyapunov function:

$$
V_{i}=V_{i-1}+\frac{\chi_{i}^{4}}{4}+\frac{1}{2 \gamma_{i}} \widetilde{\theta}_{i}^{T} \widetilde{\theta}_{i}+W_{1}
$$

where $\gamma_{i}>0$ is the design parameter.
From (43) and (44), the infinitesimal generator of $V_{i}$ can be shown as follows:

$$
\begin{aligned}
& \ell V_{i}= \ell V_{i-1}+\chi_{i}^{3} \dot{\chi}_{i}-\frac{1}{\gamma_{i}} \widetilde{\theta}_{i}^{T} \dot{\theta}_{i}+\dot{W}_{1} \\
& \leq \ell V_{i-1} \\
&+\chi_{i}^{3}\left(\alpha_{i}+\chi_{i+1}+\widetilde{\theta}_{i}^{T} \varphi\left(\widehat{x}_{i}\right)\right. \\
&-\widetilde{\theta}_{i}^{T} \varphi\left(\widehat{x}_{i}\right)+H_{i}-\frac{\partial \alpha_{i-1}}{\partial y} \\
& \times\left(e_{2}+\widetilde{\theta}_{1}^{T} \varphi\left(\widehat{x}_{1}\right)\right. \\
&+\varepsilon_{1}+\Delta_{1}(x, t)+h_{1}\left(\underline{x}_{1}\left(t-\tau_{1}(t)\right)\right) \\
&\left.\left.-\frac{1}{2} \frac{\partial^{2} \alpha_{i-1}}{\partial y^{2}} g_{1}^{T} \sigma \sigma^{T} g_{1}\right)\right) \\
&+ \frac{3}{2} \chi_{i}^{2}\left(\frac{\partial \alpha_{i-1}}{\partial y}\right)^{2} g_{1}^{T} \sigma \sigma^{T} g_{1} \\
&- \frac{1}{\gamma_{i}} \widetilde{\theta}_{i}^{T} \dot{\theta}_{i}-r W_{1}+\frac{e^{r \tau}}{2\left(1-\tau^{*}\right)} \chi_{1} H_{12}\left(\chi_{1}\right) \\
&-\frac{1}{2} \chi_{1}\left(t-\tau_{1}(t)\right) H_{12}\left(\chi_{1}\left(t-\tau_{1}(t)\right)\right) .
\end{aligned}
$$

By using Young's inequality, one can obtain

$$
\chi_{i}^{3}\left(\chi_{i+1}-\widetilde{\theta}_{i}^{T} \varphi\left(\widehat{x}_{i}\right)\right) \leq \frac{3}{4} \chi_{i}^{4}+\frac{1}{4} \chi_{i+1}^{4}+\frac{1}{2} \chi_{i}^{6}+\frac{1}{2} \widetilde{\theta}_{i}^{T} \widetilde{\theta}_{i} .
$$

Then by applying Assumptions 1 and 3, one has the following inequalities:

$$
\begin{aligned}
- & \chi_{i}^{3} \frac{\partial \alpha_{i-1}}{\partial y}\left(e_{2}+\widetilde{\theta}_{1}^{T} \varphi\left(\widehat{x}_{1}\right)+\varepsilon_{1}+\Delta_{1}(x, t)\right. \\
& \left.+h_{1}\left(\underline{x}_{1}\left(t-\tau_{1}(t)\right)\right)\right) \\
\leq & \frac{1}{2}\|e\|^{2}+\frac{5}{2}\left(\frac{\partial \alpha_{i-1}}{\partial y}\right)^{2} \chi_{i}^{6}+\frac{1}{2} \widetilde{\theta}_{1}^{T} \widetilde{\theta}_{1}+\frac{1}{2} \varepsilon_{1}^{* 2}+\frac{1}{2} \Delta_{1}^{* 2} \\
& +\frac{1}{2} \chi_{1}\left(t-\tau_{1}(t)\right) H_{12}\left(\chi_{1}\left(t-\tau_{1}(t)\right)\right)+d_{1}^{*} \\
& -\frac{1}{2} \chi_{i}^{3} \frac{\partial^{2} \alpha_{i-1}}{\partial y^{2}} g_{1}^{T} \sigma \sigma^{T} g_{1}+\frac{3}{2} \chi_{i}^{2}\left(\frac{\partial \alpha_{i-1}}{\partial y}\right)^{2} g_{1}^{T} \sigma \sigma^{T} g_{1} \\
\leq & \frac{1}{4} \chi_{i}^{6}\left(\frac{\partial^{2} \alpha_{i-1}}{\partial y^{2}}\right)^{2}+\frac{3}{4} \chi_{i}^{4}\left(\frac{\partial \alpha_{i-1}}{\partial y}\right)^{4}+|\overline{\sigma \sigma}| .
\end{aligned}
$$


Substituting (46)-(48) into (45), one has

$$
\begin{aligned}
\ell V_{i} \leq & \chi_{i}^{3}\left(\alpha_{i}+\chi_{i}+\frac{5}{2}\left(\frac{\partial \alpha_{i-1}}{\partial y}\right)^{2} \chi_{i}^{3}+\frac{1}{2} \chi_{i}^{3}\right. \\
& \left.+\frac{1}{4} \chi_{i}^{3}\left(\frac{\partial^{2} \alpha_{i-1}}{\partial y^{2}}\right)^{2}+\frac{3}{4} \chi_{i}\left(\frac{\partial \alpha_{i-1}}{\partial y}\right)^{4}+H_{i}\right) \\
& -\widetilde{\theta}_{i}^{T}\left(\frac{\dot{\theta}_{i}}{\gamma_{i}}-\varphi\left(\widehat{x}_{i}\right) \chi_{i}^{3}\right)+\frac{i-1}{2} \widetilde{\theta}_{1}^{T} \widetilde{\theta}_{1}+\frac{1}{4} \chi_{i+1}^{4} \\
& +e^{T}\left(A^{T} P+P A\right) e-c_{1} \chi_{1}^{2}-\sum_{j=2}^{i-1} c_{j} \chi_{j}^{4} \\
+ & \left(4+\frac{i}{2}+\|P\|^{2} \sum_{i=1}^{n} m_{i}^{2}\right)\|e\|^{2} \\
+ & \frac{1}{2} \sum_{j=2}^{i} \widetilde{\theta}_{j}^{T} \widetilde{\theta}_{j}+\sum_{j=1}^{i-1} \frac{\sigma_{j}}{\gamma_{j}} \widetilde{\theta}_{j}^{T} \theta_{j}+\sum_{i=1}^{n} \widetilde{\theta}_{i}^{T} \widetilde{\theta}_{i} \\
& -\frac{(n-i) \chi_{1} e^{r \tau}}{2\left(1-\tau^{*}\right)} H_{12}\left(\chi_{1}\right)-i r W_{1}+d_{i},
\end{aligned}
$$

where $d_{i}=d_{i-1}+(1 / 2) \varepsilon_{1}^{* 2}+\left|\overline{\sigma \sigma}^{T}\right|+(1 / 2) \Delta_{1}^{* 2}+d_{1}^{*}$.

Choose the intermediate control function, $\alpha_{i}$, and the adaptation law for $\theta_{i}$ as

$$
\begin{gathered}
\alpha_{i}=-c_{i} \chi_{i}-\chi_{i}-\frac{5}{2}\left(\frac{\partial \alpha_{i-1}}{\partial y}\right)^{2} \chi_{i}^{3} \\
-\frac{1}{2} \chi_{i}^{3}-\frac{1}{4} \chi_{i}^{3}\left(\frac{\partial^{2} \alpha_{i-1}}{\partial y^{2}}\right)^{2}-\frac{3}{4} \chi_{i}\left(\frac{\partial \alpha_{i-1}}{\partial y}\right)^{4}-H_{i} \\
\dot{\theta}_{i}=\gamma_{i} \varphi\left(\widehat{x}_{i}\right) \chi_{i}^{3}-\sigma_{i} \theta_{i},
\end{gathered}
$$

where $c_{i}>0$ and $\sigma_{i}>0$ are design parameters.

Substituting (50)-(51) into (49) yields

$$
\begin{aligned}
\ell V_{i} \leq & \frac{i-1}{2} \widetilde{\theta}_{1}^{T} \widetilde{\theta}_{1}+\frac{1}{2} y^{2}+\frac{1}{4} \chi_{i+1}^{4} \\
& +e^{T}\left(A^{T} P+P A\right) e+\left(4+\frac{i}{2}+\|P\|^{2} \sum_{i=1}^{n} m_{i}^{2}\right)\|e\|^{2} \\
& -c_{1} p_{1}^{2} \chi_{1}^{2}-\sum_{j=2}^{i} c_{j} \chi_{j}^{4}+\frac{1}{2} \sum_{j=2}^{i} \widetilde{\theta}_{j}^{T} \widetilde{\theta}_{j} \\
& +\sum_{j=1}^{i} \frac{\sigma_{j}}{\gamma_{1}} \widetilde{\theta}_{j}^{T} \theta_{j}+\sum_{j=1}^{n} \widetilde{\theta}_{j}^{T} \widetilde{\theta}_{j}+d_{i} .
\end{aligned}
$$

Step $n$. In the final design step, the actual control input $u_{0}$ appears. Similar to step $i$, one has

$$
\begin{aligned}
d \chi_{n}= & \left(\omega^{T} u+\widehat{f}_{n}\left(\widehat{x}_{n} \mid \theta_{n}\right)+k_{n}\left(x_{1}-\widehat{x}_{1}\right)\right) d t \\
& -\sum_{j=1}^{n-1} \frac{\partial \alpha_{n-1}}{\partial \theta_{j}} \dot{\theta}_{j} d t-\frac{\partial \alpha_{n-1}}{\partial y} d y-\sum_{j=1}^{n-1} \frac{\partial \alpha_{n-1}}{\partial \widehat{x}_{j}} d \widehat{x}_{j} \\
= & \left(\omega^{T} u+H_{n}-\frac{\partial \alpha_{n-1}}{\partial y}\right. \\
& \quad \times\left(e_{2}+\varepsilon_{1}+\widetilde{\theta}_{1}^{T} \varphi\left(\widehat{x}_{1}\right)+\Delta_{1}(x, t)+h_{1}\left(\underline{x}_{1}\left(t-\tau_{1}(t)\right)\right)\right) \\
& \left.\quad-\frac{1}{2} \frac{\partial^{2} \alpha_{n-1}}{\partial y^{2}} g_{1}^{2}(x)\right) d t-\frac{\partial \alpha_{n-1}}{\partial y} g_{1}(x) d w,
\end{aligned}
$$

where $H_{n}=\theta_{n}^{T} \varphi\left(\underline{x}_{n}\right)+\left(k_{n}-\sum_{j=1}^{n-1}\left(\partial \alpha_{n-1} / \partial \widehat{x}_{j}\right) k_{j}\right)\left(x_{1}-\right.$ $\left.\widehat{x}_{1}\right)-\sum_{j=1}^{n-1}\left(\partial \alpha_{n-1} / \partial \theta_{j}\right) \dot{\theta}_{j}-\left(\partial \alpha_{n-1} / \partial y\right)\left(\widehat{x}_{2}+\theta_{1}^{T} \varphi\left(\widehat{x}_{1}\right)\right)-$ $\sum_{j=1}^{n-1}\left(\partial \alpha_{n-1} / \partial \widehat{x}_{j}\right)\left(\widehat{x}_{j+1}+\widehat{f}_{j}\left(\underline{\hat{x}}_{j} \mid \theta_{j}\right)\right)$. Note that, by (4) and (47), one can obtain

$$
\begin{aligned}
\omega^{T} u & =\omega^{T}[\rho v+\sigma(\bar{u}-\rho v)]=\omega^{T}[\rho v+\sigma \bar{u}-\sigma \rho v] \\
& =\omega^{T}[(I-\sigma) \rho v+\sigma \bar{u}]=\sum_{j=j_{1} \cdots j_{p}} \Phi_{j} \bar{u}_{j}+\sum_{j \neq j_{1} \cdots j_{p}} \rho_{j} \varrho_{j} b_{j} u_{0} .
\end{aligned}
$$

Consider the overall Lyapunov function candidate as

$$
V_{n}=V_{n-1}+\frac{\chi_{n}^{4}}{4}+\frac{1}{2 \gamma_{n}} \widetilde{\theta}_{n}^{T} \widetilde{\theta}_{n}+W_{1},
$$

where $\gamma_{n}>0$ is the design parameter.

Design the controller $u_{0}$ and the adaptation law for $\theta_{n}$ as

$$
\begin{gathered}
u_{0}=\left(h^{\prime}\right)^{-1}\left[-c_{n} \chi_{n}-\frac{1}{4} \chi_{n}-\frac{5}{2}\left(\frac{\partial \alpha_{n-1}}{\partial y}\right)^{2} \chi_{n}^{3}-\frac{1}{2} \chi_{n}^{3}\right. \\
\left.-\frac{1}{4} \chi_{n}^{3}\left(\frac{\partial^{2} \alpha_{n-1}}{\partial y^{2}}\right)^{2}-\frac{3}{4} \chi_{n}\left(\frac{\partial \alpha_{n-1}}{\partial y}\right)^{4}-H_{n}\right] \\
\dot{\theta}_{n}=\gamma_{n} \varphi\left(\widehat{x}_{n}\right) \chi_{n}^{3}-\sigma_{n} \theta_{n},
\end{gathered}
$$

where $\sigma_{n}>0$ and $c_{n}>0$ are design parameters, and $h^{\prime}=$ $\sum_{j \neq j_{1} \ldots j_{p}} \rho_{j} \oplus_{j} b_{j}$.

Substituting (52)-(56) into (51), one can obtain

$$
\begin{aligned}
\ell V_{n} \leq & -e^{T} Q e-c_{1} p_{1}^{2} \chi_{1}^{2}-\sum_{j=2}^{n} c_{j} \chi_{j}^{4} \\
& +\frac{n-1}{2} \widetilde{\theta}_{1}^{T} \widetilde{\theta}_{1}+\frac{1}{2} \sum_{j=2}^{n} \widetilde{\theta}_{j}^{T} \widetilde{\theta}_{j} \\
& +\sum_{j=1}^{n} \frac{\sigma_{j}}{\gamma_{j}} \widetilde{\theta}_{j}^{T} \theta_{j}+\sum_{j=1}^{n} \widetilde{\theta}_{j}^{T} \widetilde{\theta}_{j}-n r W_{1}+d_{n},
\end{aligned}
$$

where $d_{n}=d_{n-1}+(1 / 2) \varepsilon_{1}^{* 2}+\left|\overline{\sigma \sigma}^{T}\right|+(1 / 2) \Delta_{1}^{* 2}+d_{1}^{*}$. 
By completing the square for each parameter estimate,

$$
\frac{\sigma_{j}}{\gamma_{j}} \widetilde{\theta}_{j}^{T} \theta_{j} \leq-\frac{1}{2} \frac{\sigma_{j}}{\gamma_{j}} \widetilde{\theta}_{j}^{T} \widetilde{\theta}_{j}+\frac{1}{2} \frac{\sigma_{j}}{\gamma_{j}} \theta_{j}^{* T} \theta_{j}^{*} .
$$

Substituting (58)-(60) into (57) yields

$$
\begin{aligned}
\ell V_{n} \leq & -q_{n}\|e\|^{2}-c_{1} p_{1}^{2} \chi_{1}^{2}-\sum_{j=2}^{n} c_{j} \chi_{j}^{4} \\
& -\frac{\sigma_{1} / \gamma_{1}-n-1}{2} \widetilde{\theta}_{1}^{T} \widetilde{\theta}_{1}-\frac{1}{2} \sum_{j=2}^{n}\left(\frac{\sigma_{j}}{\gamma_{j}}-3\right) \widetilde{\theta}_{j}^{T} \widetilde{\theta}_{j} \\
& -n r W_{1}+d,
\end{aligned}
$$

where $q_{n}=\lambda_{\min }(Q), d=d_{n}+(1 / 2) \sum_{j=1}^{n}\left(\sigma_{j} / \gamma_{j}\right) \theta_{j}^{* T} \theta_{j}^{*}$.

Define $c=\min \left\{q_{n} / \lambda_{\max }(P), 2 c_{1} p_{1}^{2}, 4 c_{j},\left(\left(\sigma_{1} / \gamma_{1}\right)-n-\right.\right.$ 1) $\left.\gamma_{1},\left(\left(\sigma_{j} / \gamma_{j}\right)-3\right), n r\right\}$.

Then (61) can be rewritten as

$$
\ell V_{n} \leq-c V_{n}+d
$$

Multiplying $V$ by $e^{C t}$ and by Itô formula leads to

$$
d\left(e^{C t} V\right)=e^{C t}(C V+\ell V) d t+e^{C t} \sum_{i=1}^{n} M_{i} d w,
$$

where $M_{i}=\partial V / \partial \chi_{i}\left(-\left(\partial \alpha_{i-1} / \partial y\right) g_{1}(x)\right)$ for $i=2, \ldots, n$, and $M_{1}=g_{1}(x)$.

From (61) and (62), one has

$$
d\left(e^{C t} V\right) \leq e^{C t} D d t+e^{C t} \sum_{i=1}^{n} M_{i} d w
$$

Integrating (63) over $[0, T]$, we get

$$
V(T) \leq e^{-C T} V(0)+\frac{D}{C}+e^{-C T} \int_{0}^{T} e^{C s} \Omega_{1} d w(s) .
$$

Taking expectation on (64), it follows that

$$
E[V(T)] \leq V(0)+\frac{D}{C}
$$

where $V(0)=(1 / 2) e^{T}(0) P e(0)+(1 / 2) \chi_{1}^{2}(0)+\sum_{i=2}^{n}(1 / 4) \chi_{i}^{4}(0)+$ $\sum_{i=1}^{n}(1 / 2) \gamma_{i} \widetilde{\theta}_{i}^{T}(0) \widetilde{\theta}_{i}(0)$.

By (65) and using the similar arguments in [8-11], it follows that all the signals of the closed-loop system are bounded in probability.

Theorem 7. For nonlinear system (1), if Assumptions 14 are satisfied, the controller (56) with the state observer (24), the intermediate control functions (40) and (50), and parameter adaptive laws (41), (51), and (57) can guarantee that all the signals in the closed-loop system are bounded in probability, and the output error converges to the given prescribed performance bounds.

\section{Simulation Study}

In this section, an example is given to illustrate the effectiveness of the proposed adaptive fuzzy FTC method.

Example 8. Consider the following:

$$
\begin{aligned}
& d x_{1}=\left(x_{2}+f_{1}\left(x_{1}\right)+\Delta_{1}(x, t)\right. \\
& \left.+h_{1}\left(x_{1}\left(t-\tau_{1}(t)\right)\right)\right) d t+g_{1}(x) d w, \\
& d x_{2}=\left(\omega^{T} u+f_{2}\left(\underline{x}_{2}\right)+\Delta_{2}(x, t)\right. \\
& \left.+h_{2}\left(\underline{x}_{2}\left(t-\tau_{2}(t)\right)\right)\right) d t+g_{2}(x) d w, \\
& y=x_{1}
\end{aligned}
$$

where $f_{1}\left(x_{1}\right)=x_{1} e^{-0.5 x_{1}^{2}}, f_{2}\left(\underline{x}_{2}\right)=\sin \left(0.5 x_{1} x_{2}\right), \Delta_{1}(x, t)=$ $\sin x_{1}, \Delta_{2}(x, t)=\cos \left(x_{1} x_{2}\right), g_{1}(x)=\sin x_{1}^{2}, g_{2}(x)=$ $x_{1}^{2} \cos x_{2}, \omega_{1}=\omega_{2}=1, h_{1}=\left(x_{1}\left(t-\tau_{1}(t)\right)\right) /\left(1+x_{1}^{2}\left(t-\tau_{1}(t)\right)\right)$, $\tau_{1}(t)=0.5 \sin (t), h_{2}=\left(x_{1}\left(t-\tau_{2}(t)\right) \sin \left(x_{2}\left(t-\tau_{2}(t)\right)\right)\right) /(1+$ $\left.x_{1}^{4}\left(t-\tau_{2}(t)\right)\right), \tau_{2}(t)=0.5 \sin (t)$, and $u=\left[u_{1}, u_{2}\right] . \dot{w}(t)$ is assumed to be a Gaussian white noise with $\sigma(t)=1$. The tracking reference signal is chosen as $y_{r}(t)=\sin (t)$.

The actuator faults are $\bar{u}_{1}=65$, when $t \geq 2$, and $u_{2}=$ $0.8 v_{2}$, when $t \geq 2$.

Choose fuzzy membership functions as

$$
\mu_{F_{i}^{l}}\left(\widehat{x}_{i}\right)=\exp \left[-\frac{\left(\widehat{x}_{i}-3+l\right)^{2}}{16}\right], \quad l=1,2,3,4,5 .
$$

Construct the FLSs $\widehat{f}_{i}\left(\underline{\widehat{x}}_{i} \mid \theta_{i}\right)=\theta_{i}^{T} \varphi_{i}\left(\underline{\widehat{x}}_{i}\right)$ to approximate the unknown nonlinear function $f_{i}(\cdot), i=1,2$.

The parameters of the prescribed performance functions are selected as $\mu_{1,0}=1.5, \mu_{1, \infty}=0.05, n_{1}=1, \delta_{1 \text { min }}=0.4$, $\delta_{1 \max }=0.45$, and $\mu_{1}(t)=1.45 e^{-t}+0.05$. (20) is

Setting the parameters $k_{1}=k_{2}=1.4$, the state observer

$$
\begin{aligned}
& \dot{\hat{x}}_{1}=\widehat{x}_{2}+\widehat{f}_{1}\left(\widehat{x}_{1} \theta_{1}\right)+1.4\left(x_{1}-\widehat{x}_{1}\right), \\
& \dot{\hat{x}}_{2}=u_{1}+u_{2}+\widehat{f}_{2}\left(\widehat{x}_{1}, \widehat{x}_{2} \theta_{2}\right)+1.4\left(x_{1}-\widehat{x}_{1}\right), \\
& \hat{y}=\widehat{x}_{1} .
\end{aligned}
$$

The design parameters in the controllers $u_{0}(56)$, the intermediate controls function $\alpha_{1}(40)$, and the adaptive laws $\theta_{1}(41)$ and $\theta_{2}$ (57) are selected as $c_{1}=55, c_{2}=15, \gamma_{1}=\gamma_{2}=0.01$, $\rho_{1}=\rho_{2}=1, b_{1}\left(\widehat{x}_{1}\right)=b_{2}\left(\underline{x}_{2}\right)=1$, and $\sigma_{1}=\sigma_{2}=0.1$.

The initial conditions are chosen as follows: $x_{1}(0)=0.1$, $x_{2}(0)=0, \widehat{x}_{1}(0)=\widehat{x}_{2}(0)=0, \theta_{1}^{T}(0)=[0,0,0,0,0]$, and $\theta_{2}^{T}(0)=[0,0,0,0,0]$.

Applying the control method in this paper to control (66), the simulation results are shown by Figures 1, 2, 3, 4, and 5, respectively. From Figures 1-5, it is shown that the proposed adaptive FTC method can guarantee that all the variables are bounded in probability, the output $y(t)$ can track the given reference signal $y_{r}$, and the tracking error remains within the prescribed performance bounds. 


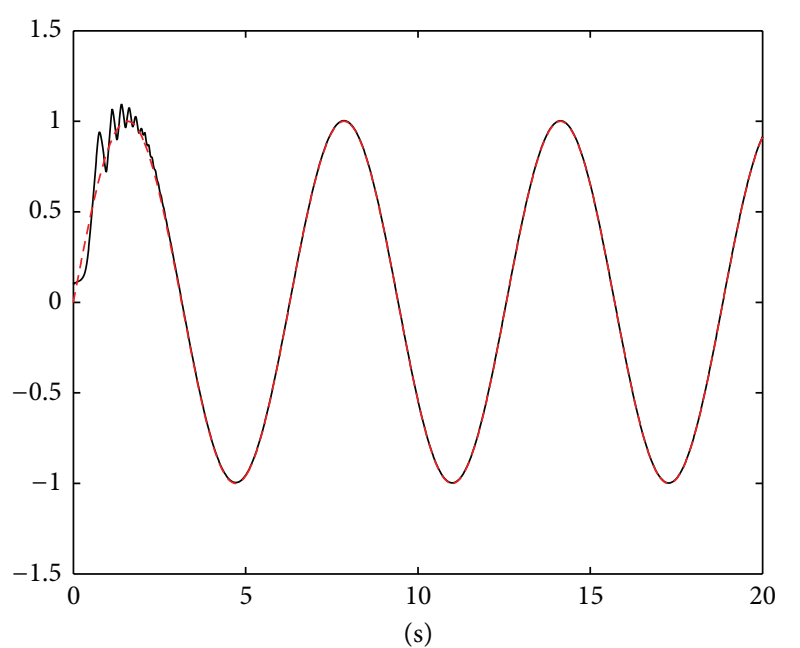

Figure 1: The curves of $y_{1}$ (solid) and $y_{r}$ (dash).

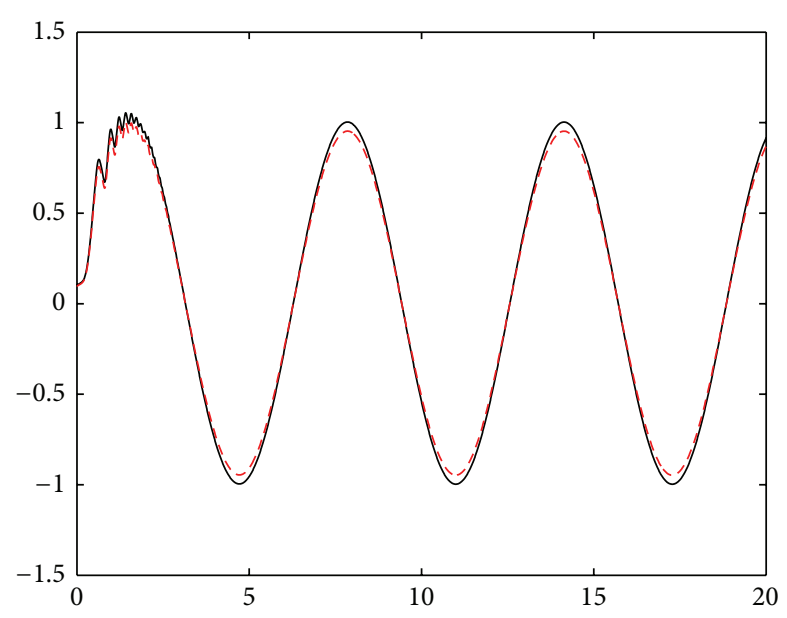

(s)

FIgURE 2: The curves of $x_{1}$ (solid) and its estimation $\widehat{x}_{1}$ (dash).

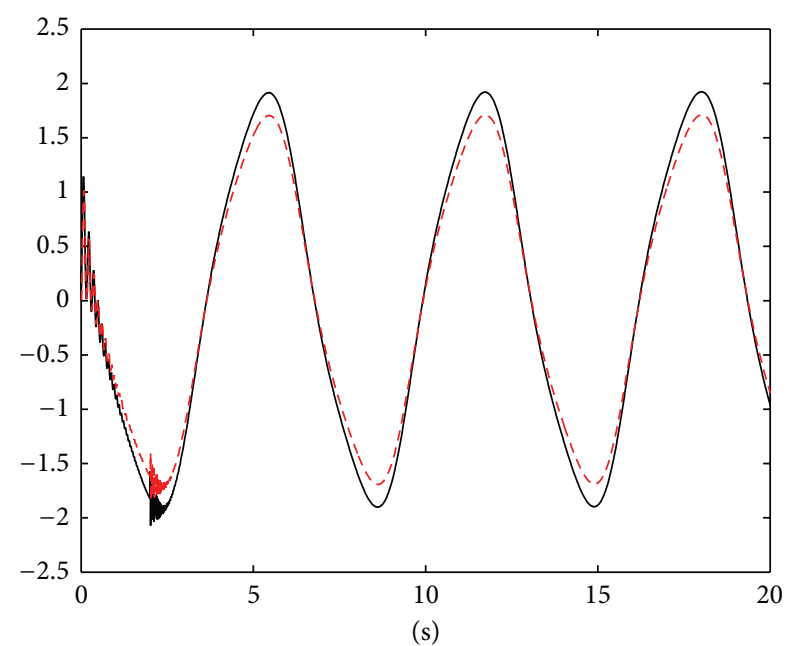

FIgUre 3: The curves of $x_{2}$ (solid) and its estimation $\widehat{x}_{2}$ (dash).

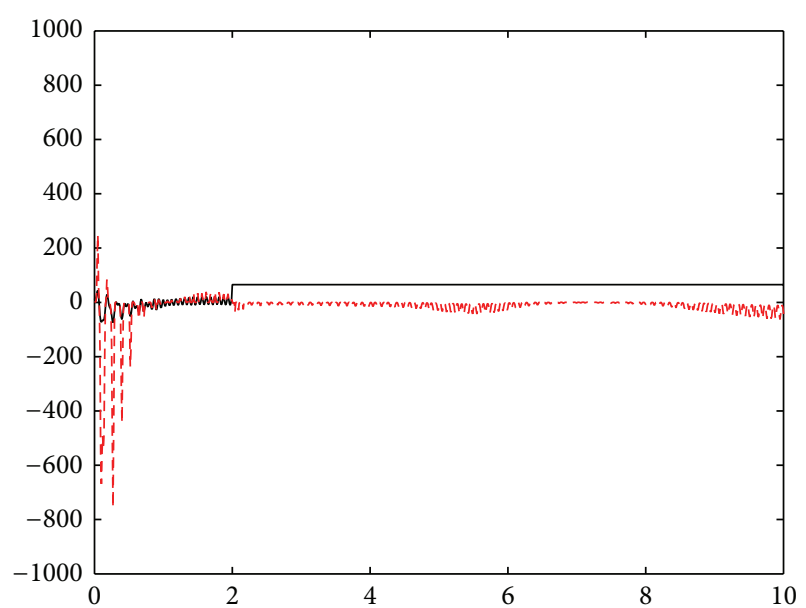

(s)

FIgURE 4: The curves of $u_{1}$ (solid) and $u_{2}$ (dash).

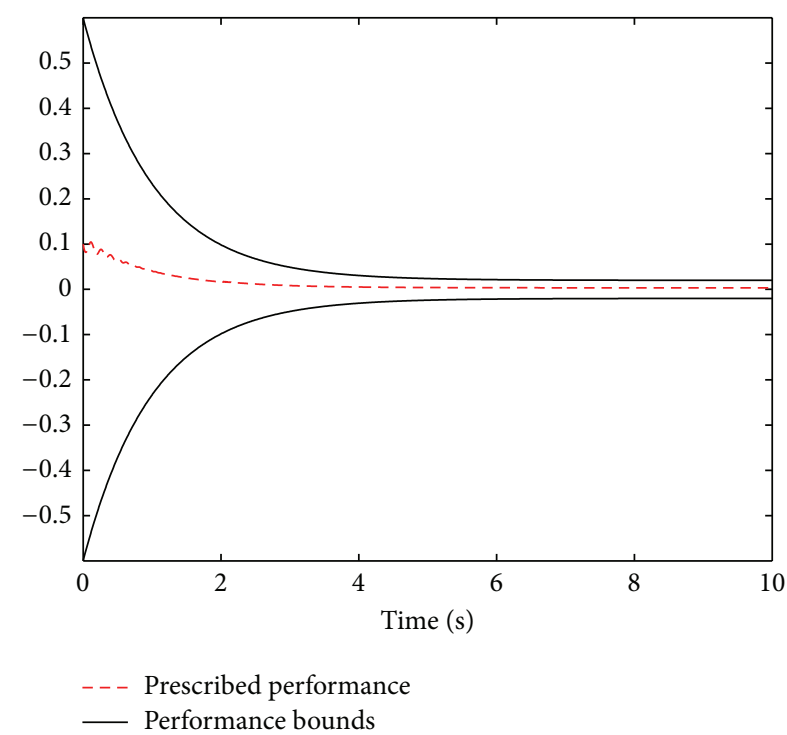

Figure 5: The curves of $z_{1}$ and performance bounds.

\section{Conclusion}

This paper has developed an adaptive fuzzy FTC method for a class of uncertain stochastic nonlinear systems with unmeasured states, unknown time-delay, actuator faults, and tracking error constrained. The considered faults are modeled as both loss of effectiveness and lock-in-place. With the help of fuzzy logic systems to approximate the unknown nonlinear functions, a fuzzy adaptive observer has been developed for estimating the unmeasured states. Combining the backstepping technique with the nonlinear tolerant-fault control theory, a novel adaptive fuzzy FTC approach has been constructed. It has proved that the proposed control approach can guarantee that all the signals of the resulting closed-loop system are bounded in probability, and the tracking error converges to an adjustable neighborhood of the origin and remains within the prescribed performance bounds. Future 
research will concentrate on the adaptive FTC methods for uncertain MIMO stochastic nonlinear systems and uncertain stochastic nonlinear large-scale systems based on the results of this paper and the references of [26-36].

\section{Appendix}

Proof. From (25), one can obtain

$$
\begin{aligned}
\ell V_{0} \leq & e^{T}\left(A^{T} P+P A\right) e \\
& +2 e^{T} P\left(\varepsilon+\Delta f+\Delta+h+\sum_{i=1}^{n} B_{i} \widetilde{\theta}_{i}^{T} \varphi_{i}\left(\underline{\widehat{x}}_{i}\right)\right) \\
& +\operatorname{Tr}\left[\sigma g^{T} P g \sigma^{T}\right],
\end{aligned}
$$

where $\lambda_{\min }(Q)$ is the smallest eigenvalue of matrix $Q, \Delta f=$ $\left[\Delta f_{1}, \ldots, \Delta f_{n}\right]^{T}, \varepsilon=\left[\varepsilon_{1}, \ldots, \varepsilon_{n}\right]^{T}, \Delta=\left[\Delta_{1}, \ldots, \Delta_{n}\right]^{T}, h=$ $\left[h_{1}, \ldots, h_{n}\right]^{T}$, and $\widetilde{\theta}_{i}=\theta_{i}^{*}-\theta_{i}$.

By using Young's inequality and Assumption 1, one has the following inequalities:

$$
\begin{gathered}
\operatorname{Tr}\left[\sigma g^{T} P g \sigma^{T}\right] \leq \frac{1}{2}\|P\|^{2}+\frac{1}{2}\left|\overline{\sigma \sigma}^{T}\right|^{2} \\
2 e^{T} P(\varepsilon+\Delta f+\Delta+h) \leq 4\|e\|^{2}+\|P\|^{2}\left\|\varepsilon^{*}\right\|^{2} \\
+\|P\|^{2}\left\|\Delta^{*}\right\|^{2}+\|P\|^{2}\|\Delta f\|^{2} \\
+\sum_{i=1}^{n} \chi_{1}\left(t-\tau_{i}(t)\right) H_{i 2}\left(\chi_{1}\left(t-\tau_{i}(t)\right)\right)+\sum_{i=1}^{n} d_{i}^{*} \\
\leq\left(4+\|P\|^{2} \sum_{i=1}^{n} m_{i}^{2}\right)\|e\|^{2}+\|P\|^{2}\left(\left\|\varepsilon^{*}\right\|^{2}+\left\|\Delta^{*}\right\|^{2}\right) .
\end{gathered}
$$

By using Young's inequality and the fact $\varphi_{i}^{T}\left(\widehat{x}_{i}\right) \varphi_{i}\left(\widehat{x}_{i}\right) \leq 1$, one has

$$
\begin{aligned}
& 2 e^{T} P \sum_{i=1}^{n} B_{i} \widetilde{\theta}_{i}^{T} \varphi_{i}\left(\underline{\underline{x}}_{i}\right) \\
& \quad \leq e^{T} P P^{T} e+\sum_{i=1}^{n} \widetilde{\theta}_{i}^{T} \varphi_{i}^{T}\left(\underline{\widehat{x}}_{i}\right) \varphi_{i}\left(\underline{\widehat{x}}_{i}\right) \widetilde{\theta}_{i} \\
& \quad \leq \lambda_{\max }^{2}(P)\|e\|^{2}+\sum_{i=1}^{n} \widetilde{\theta}_{i}^{T} \widetilde{\theta}_{i} .
\end{aligned}
$$

Substituting (A.2)-(A.4) into (A.1) yields (27).

\section{Conflict of Interests}

The authors declare that there is no conflict of interests regarding the publication of this paper.

\section{Acknowledgments}

This work was supported by the National Natural Science Foundation of China (nos. 61374113, 61074014, and 61203008) and Program for Liaoning Innovative Research Team in University (LT2012013).

\section{References}

[1] Y.-C. Wang, H.-G. Zhang, and Y.-Z. Wang, "Fuzzy adaptive control of stochastic nonlinear systems with unknown virtual control gain function," Acta Automatica Sinica, vol. 32, no. 2, pp. 170-178, 2006.

[2] H. Wang, B. Chen, X. Liu, K. Liu, and C. Lin, "Adaptive neural tracking control for stochastic nonlinear strict-feedback systems with unknown input saturation," Information Sciences, vol. 269, pp. 300-315, 2014.

[3] H. Wang, B. Chen, and C. Lin, "Adaptive fuzzy control for purefeedback stochastic nonlinear systems with unknown deadzone input," International Journal of Systems Science, 2013.

[4] Z. X. Yu, Z. H. Jin, and H. B. Du, "Adaptive neural control for a class of non-affine stochastic non-linear systems with timevarying delay: A Razumikhin-Nussbaum method," IET Control Theory and Applications, vol. 6, no. 1, pp. 14-23, 2012.

[5] Z. X. Yu, S. G. Li, and H. B. Du, "Razumikhin-Nussbaumlemma-based adaptive neural control for uncertain stochastic pure-feedback nonlinear systems with time-varying delays," International Journal of Robust and Nonlinear Control, vol. 23, no. 11, pp. 1214-1239, 2013.

[6] W. Chen, L. Jiao, J. Li, and R. Li, "Adaptive NN backstepping output-feedback control for stochastic nonlinear strictfeedback systems with time-varying delays," IEEE Transactions on Systems, Man, and Cybernetics, Part B: Cybernetics, vol. 40, no. 3, pp. 939-950, 2010.

[7] W. S. Chen, L. C. Jiao, and Z. B. Du, "Output-feedback adaptive dynamic surface control of stochastic non-linear systems using neural network," IET Control Theory \& Applications, vol. 4, no. 12, pp. 3012-3021, 2010.

[8] S. C. Tong, Y. Li, Y. M. Li, and Y. J. Liu, "Observer-based adaptive fuzzy backstepping control for a class of stochastic nonlinear strict-feedback systems," IEEE Transactions on Systems, Man and Cybernetics, Part B, vol. 41, no. 6, pp. 1693-1704, 2011.

[9] S. C. Tong, T. Wang, Y. M. Li, and B. Chen, "A combined backstepping and stochastic small-gain approach to robust adaptive fuzzy output feedback control," IEEE Transactions on Fuzzy Systems, vol. 21, no. 2, pp. 314-327, 2013.

[10] J. Li, W. Chen, and J.-M. Li, "Adaptive NN output-feedback decentralized stabilization for a class of large-scale stochastic nonlinear strict-feedback systems," International Journal of Robust and Nonlinear Control, vol. 21, no. 4, pp. 452-472, 2011.

[11] S. C. Tong, S. Sui, and Y. M. Li, "Adaptive fuzzy decentralized output stabilization for stochastic nonlinear large-scale systems with unknown control directions," IEEE Transactions on Fuzzy Systems, 2013.

[12] P. Li and G. Yang, "Backstepping adaptive fuzzy control of uncertain nonlinear systems against actuator faults," Journal of Control Theory and Applications, vol. 7, no. 3, pp. 248-256, 2009.

[13] P. Li and G.-H. Yang, "Adaptive fuzzy control of unknown nonlinear systems with actuator failures for robust output tracking," in Proceedings of the American Control Conference (ACC '08), pp. 4898-4903, June 2008.

[14] P. Li and G. Yang, "An adaptive fuzzy design for fault-tolerant control of MIMO nonlinear uncertain systems," Journal of Control Theory and Applications, vol. 9, no. 2, pp. 244-250, 2011. 
[15] S. Tong, B. Huo, and Y. Li, "Observer-based adaptive decentralized fuzzy fault-tolerant control of nonlinear large-scale systems with actuator failures," IEEE Transactions on Fuzzy Systems, vol. 22, no. 1, pp. 1-15, 2014.

[16] Q. K. Shen, B. Jiang, and V. Cocquempot, "Fuzzy logic systembased adaptive fault-tolerant control for near-space vehicle attitude dynamics with actuator faults," IEEE Transactions on Fuzzy Systems, vol. 21, no. 2, pp. 289-300, 2013.

[17] S. C. Tong, T. Wang, and Y. M. Li, "Fuzzy adaptive actuator failure compensation control of uncertain stochastic nonlinear systems with unmodeled dynamics," IEEE Transactions on Fuzzy Systems, vol. 22, no. 3, pp. 563-574, 2014.

[18] C. P. Bechlioulis and G. A. Rovithakis, "Robust adaptive control of feedback linearizable MIMO nonlinear systems with prescribed performance," IEEE Transactions on Automatic Control, vol. 53, no. 9, pp. 2090-2099, 2008.

[19] T. Wang, Y. F. Zhang, J. B. Qiu, and H. J. Gao, "Adaptive fuzzy backstepping control for a class of nonlinear systems with sampled and delayed measurements," vol. PP, no. 99, p. 1, 2014.

[20] C. Hua, L. Zhang, and X. Guan, "Output feedback control for interconnected time-delay systems with prescribed performance," Neurocomputing, vol. 129, pp. 208-215, 2014.

[21] C. P. Bechlioulis and G. A. Rovithakis, "Prescribed performance adaptive control for multi-input multi-output affine in the control nonlinear systems," IEEE Transactions on Automatic Control, vol. 55, no. 5, pp. 1220-1226, 2010.

[22] H.-B. Ji and H.-S. Xi, "Adaptive output-feedback tracking of stochastic nonlinear systems," IEEE Transactions on Automatic Control, vol. 51, no. 2, pp. 355-360, 2006.

[23] L. X. Wang, Adaptive Fuzzy Systems and Control, Prentice Hall, Englewood Cliffs, NJ, USA, 1994.

[24] S. C. Tong and Y. M. Li, "Adaptive fuzzy output feedback tracking backstepping control of strict-feedback nonlinear systems with unknown dead zones," IEEE Transactions on Fuzzy Systems, vol. 20, no. 1, pp. 168-180, 2012.

[25] S. Tong, C. L. Liu, and Y. M. Li, "Fuzzy-adaptive decentralized output-feedback control for large-scale nonlinear systems with dynamical uncertainties," IEEE Transactions on Fuzzy Systems, vol. 18, no. 5, pp. 845-861, 2010.

[26] S. Yin, X. Yang, and H. R. Karimi, "Data-driven adaptive observer for fault diagnosis," Mathematical Problems in Engineering, vol. 2012, Article ID 832836, 21 pages, 2012.

[27] S. Yin, S. Ding, X. Xie, and H. Luo, "A review on basic datadriven approaches for industrial process monitoring," IEEE Transactions on Industrial Electronics, vol. 61, no. 11, pp. 64186428, 2014.

[28] H. Y. Li, X. J. Jing, H. K. Lam, and P. Shi, "Fuzzy sampleddata control for uncertain vehicle suspension systems," IEEE Transactions on Cybernetics, vol. 44, no. 7, pp. 1111-1126, 2014.

[29] S. Yin, X. Li, H. Gao, and O. Kaynak, "Data-based techniques focused on modern industry: an overview," IEEE Transactions on Industrial Electronics, 2014.

[30] H. Li, X. Jing, and H. R. Karimi, "Output-feedback-based $H_{\infty}$ control for vehicle suspension systems with control delay," IEEE Transactions on Industrial Electronics, vol. 61, no. 1, pp. 436-446, 2014.

[31] S. Yin, G. Wang, and H. R. Karimi, "Data-driven design of robust fault detection system for wind turbines," Mechatronics, vol. 24, no. 4, pp. 298-306, 2014.

[32] Z. G. Feng, J. Lam, and H. J. Gao, " $\alpha$-dissipativity analysis of singular time-delay systems," Automatica, vol. 47, no. 11, pp. 2548-2552, 2011.
[33] H. Li, H. Gao, P. Shi, and X. Zhao, "Fault-tolerant control of Markovian jump stochastic systems via the augmented sliding mode observer approach," Automatica, vol. 50, no. 7, pp. 18251834, 2014.

[34] Z. Feng, J. Lam, and G.-H. Yang, "Optimal partitioning method for stability analysis of continuous/discrete delay systems," International Journal of Robust and Nonlinear Control, 2013.

[35] S. Yin, G. Wang, and X. Yang, "Robust PLS approach for KPIrelated prediction and diagnosis against outliers and missing data," International Journal of Systems Science, vol. 45, no. 7, pp. 1375-1382, 2014.

[36] Z. Q. Zhang, S. Y. Xu, and B. Y. Zhang, "Asymptotic tracking control of uncertain nonlinear systems with unknown actuator nonlinearity," IEEE Transactions on Automatic Control, vol. 59, no. 5, pp. 1336-1341, 2014. 


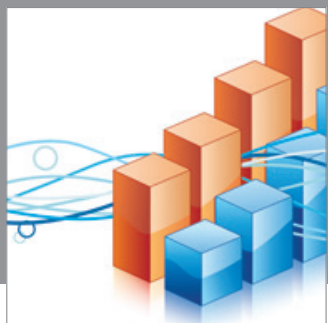

Advances in

Operations Research

mansans

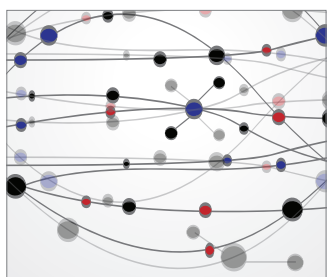

The Scientific World Journal
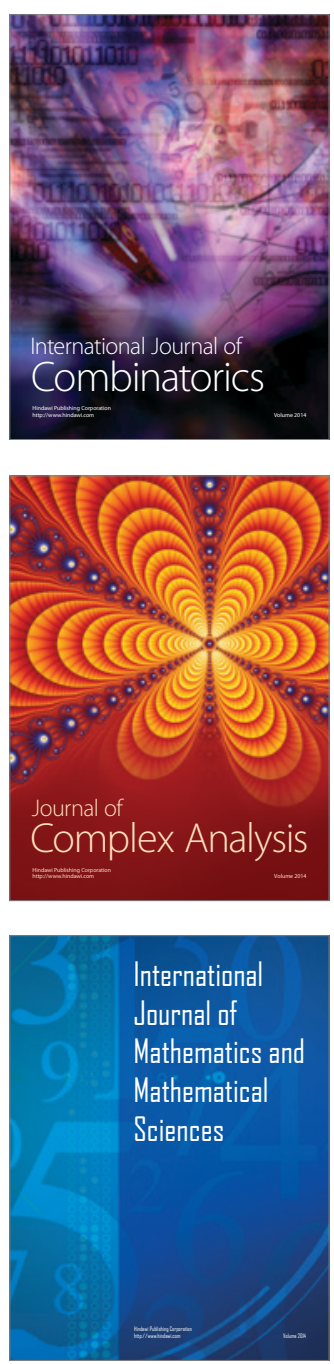
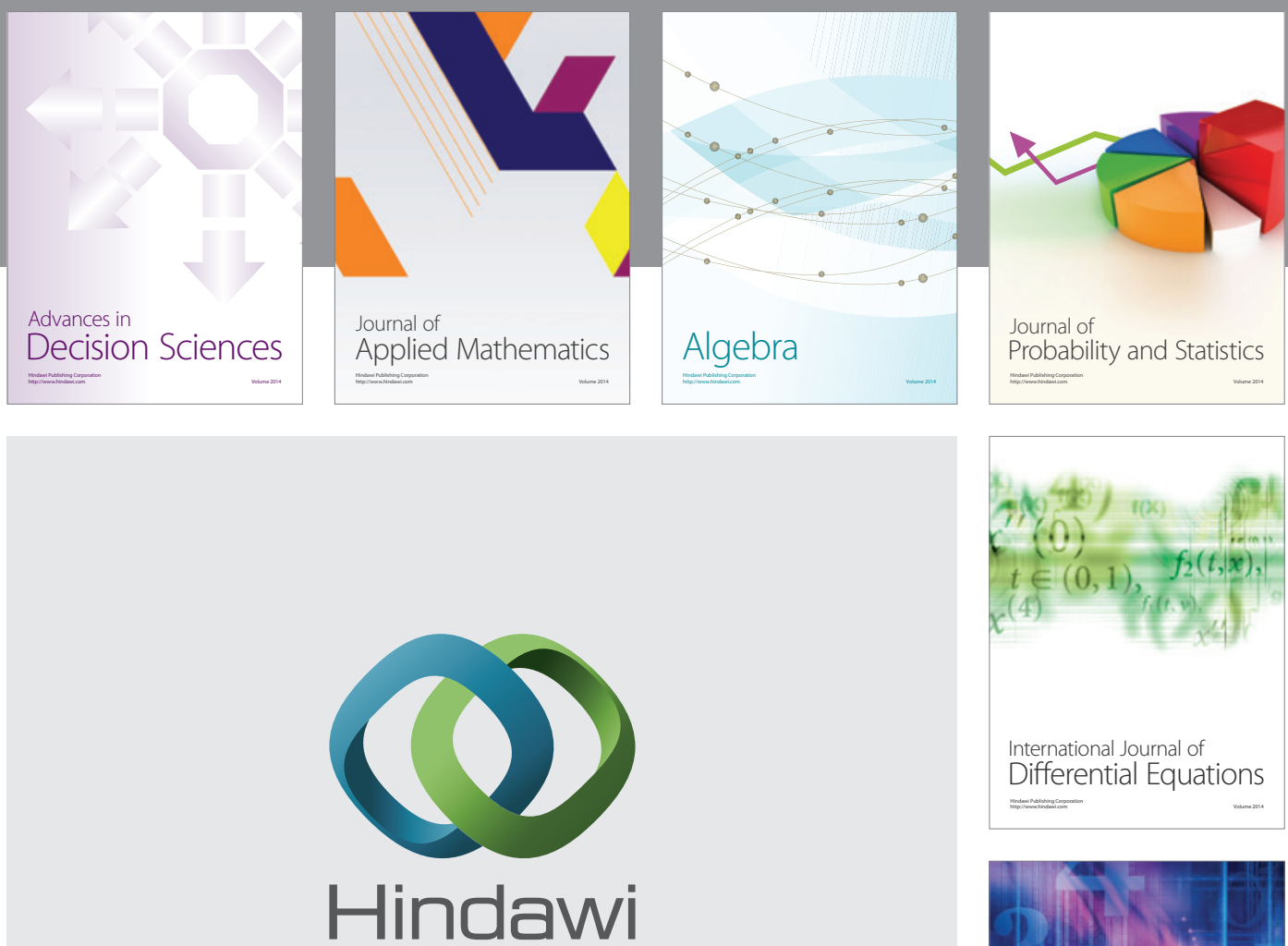

Submit your manuscripts at http://www.hindawi.com
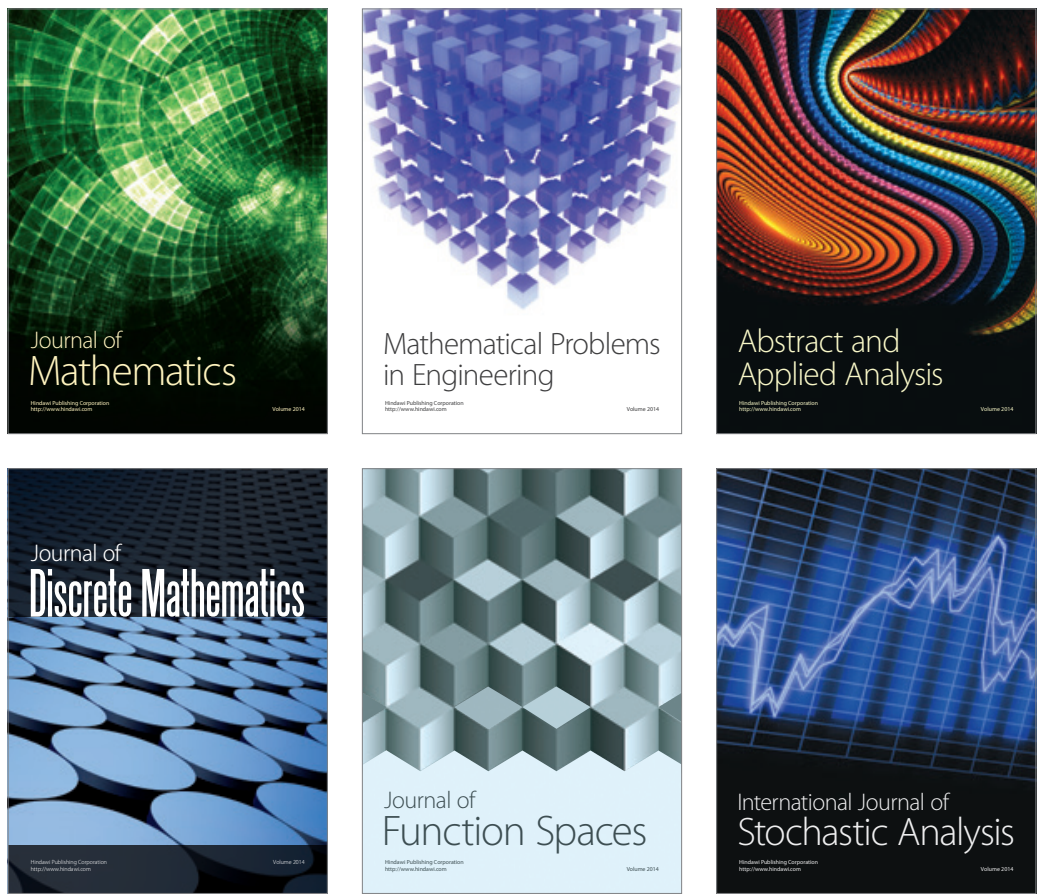

Journal of

Function Spaces

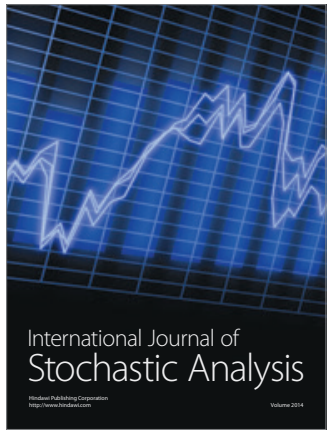

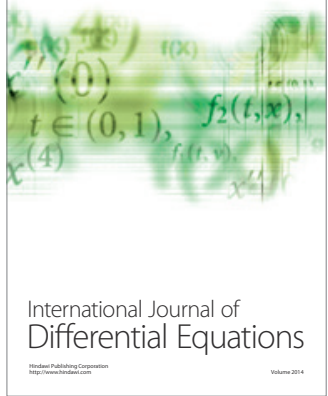
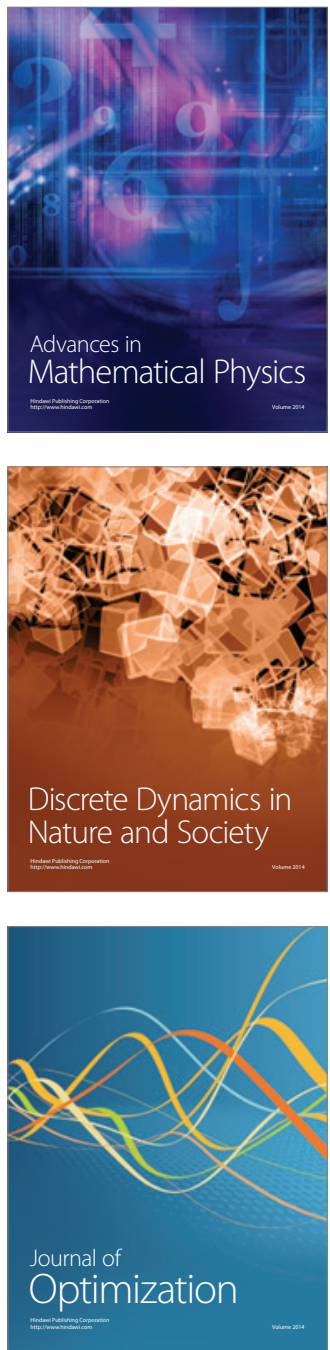